\title{
El concepto de vulnerabilidad macroeconómica. Una revisión de la literatura
}

\section{The concept of macroeconomic vulnerability. A review of the literature}

\author{
Luis Hernando Portillo-Riascos \\ Universidad de Nariño, San Juan de Pasto, Colombia \\ luihero2@ucm.es \\ https://orcid.org/0000-0003-3362-6934
}

Recibido: 07-06-19

Aprobado: 12-02-20

\footnotetext{
* Este artículo hace parte de una investigación titulada "Vulnerabilidad e inestabilidad macroeconómica de Colombia en el periodo 1990-2015", cuya memoria fue presentada para optar al título de Doctor en Economía por la Universidad Complutense de Madrid (año 2019).
}

1 Doctor en Economía. 


\section{Resumen}

El objetivo de este estudio es hacer una revisión del significado y los alcances que tiene el concepto de vulnerabilidad macroeconómica (VM), enfatizando en la situación a la cual se enfrentan las economías en desarrollo. Para este propósito, se realizó una revisión bibliográfica de las publicaciones más relevantes acerca de este tema. Como resultado de este estudio, se identificaron tres enfoques que abordan el estudio de la VM: a) el estructural; b) el que asocia la VM con la ocurrencia de episodios de crisis; y c) aquel que plantea que la VM está relacionada con la inestabilidad macroeconómica real. Más allá de los contrastes que se encontraron entre las diferentes posturas teóricas, se concluye que la VM es un fenómeno muy arraigado en las economías latinoamericanas, cuyas consecuencias se han agravado como resultado de la profundización de la integración comercial y financiera que se ha dado en las últimas décadas.

Palabras clave: vulnerabilidad macroeconómica; shocks externos; economías en desarrollo; neoestructuralismo; macroeconomía del desarrollo.

Clasificación JEL: F41, O54, O23, O11.

\section{Abstract}

The objective of this study is to review the meaning and scope of the concept of macroeconomic vulnerability (MV), emphasizing the situation faced by developing economies. For this purpose, a literature review of the most relevant publications on this topic was carried out. As a result of this study, three approaches were identified that address the VM study: (a) the structural one; (b) the one that associates the VM with the occurrence of crisis episodes; and (c) the one that argues that the VM is related to real macroeconomic instability. Beyond the contrasts that were found between the different theoretical positions, it is concluded that the VM is a phenomenon deeply rooted in the Latin American economies, whose consequences have worsened as a result of the deepening of trade and financial integration that has taken place in recent decades.

Keywords: macroeconomic vulnerability; external shocks; developing economies; neo-structuralism; development macroeconomics.

JEL classification: F41, O54, O23, O11.

Portillo-Riascos, L. H. (2020). El concepto de vulnerabilidad macroeconómica. Una revisión de la literatura. Sociedad y economía, (40), 99-118. https://doi.org/10.25100/sye.voi40.8020 


\section{Introducción}

El objetivo de este estudio es hacer una revisión de la literatura acerca del significado y los alcances que tiene el concepto de vulnerabilidad, enfatizando en dos aspectos: la dimensión económica del fenómeno y la situación a la cual se enfrentan los países en desarrollo; particularmente los latinoamericanos.

La necesidad de estudiar la VM se explica por dos razones: a) porque este es un concepto muy amplio, que se utiliza desde distintas disciplinas, enfoques, organizaciones y contextos; y b) porque no hay pleno acuerdo en sus alcances e implicaciones (Chambers, 1989; Busso, 2001; Villagrán, 2006; Seth y Ragab, 2012). Dicha circunstancia también ha llevado a que se propongan numerosos métodos para la cuantificación de la VM (Kaminsky, Lizondo y Reinhart, 1998; FMI, 1998; Briguglio, 2014; Angeon y Bates, 2015; Guillaumont, 2016).

A partir de los argumentos señalados, esta investigación tiene como propósito sintetizar y contrastar las diferentes posturas teóricas desde las cuales se ha abordado el estudio de la VM. En concreto, se hace referencia a tres enfoques que se han venido utilizando para el estudio de la VM, que son: a) el estructural; b) aquel que asocia la VM con la ocurrencia de cierto tipo de episodios de crisis (financiera o real); y c) el enfoque en el que la VM tiene que ver con la prevalencia de la inestabilidad macroeconómica real.

Desde la perspectiva metodológica, inicialmente, se identifican las semejanzas y las divergencias entre las diferentes posturas teóricas, lo cual contribuye a clarificar las características del fenómeno estudiado. Posteriormente, se resaltan los aportes realizados desde el Estructuralismo Latinoamericano y el Neoestructuralismo, en los cuales -a pesar de no existir una propuesta formal para abordar el estudio de la VM- se concluye que hay un conjunto coherente de elementos que permiten su articulación.

Este artículo está organizado en tres epígrafes, incluida la introducción. En el segundo epígrafe, se presenta la revisión de la literatura acerca de los diferentes enfoques a partir de los cuales se ha abordado el estudio de la VM. Para este propósito, inicialmente, se hace una exploración conceptual; posteriormente, se aborda el análisis de los tipos de vulnerabilidades $\mathrm{y}$, por último, se sistematizan y contrastan los tres enfoques de estudio de la VM, identificados en esta investigación. En el tercer epígrafe, se relacionan las principales conclusiones de este estudio.

\section{Los enfoques de la VM}

\subsection{Concepto}

En términos generales, la vulnerabilidad se define como la predisposición o susceptibilidad de un sujeto o colectivo para verse afectados por un evento externo en un instante del tiempo; y la incapacidad de los mismos para asumir los daños, una vez haya tenido lugar dicho acontecimiento (Villagrán, 2006; Angeon y Bates, 2015; Guillaumont, 2016). En esta definición, la primera parte corresponde estrictamente a la vulnerabilidad, mientras que la segunda hace alusión a la resiliencia, tal y como se expone más adelante (Briguglio, 1995; Briguglio, Cordina, Farrugia y Vella, 2009; Guillaumont, 2010).

A partir de la diferencia entre vulnerabilidad y resiliencia, Chambers (1989) señala que la vulnerabilidad se puede agrupar de la siguiente manera: la exposición a ciertas contingencias y la dificultad para cubrirlas. El primer componente tiene que ver con el carácter externo de la vulnerabilidad, asociado a la exposición de los sujetos a presiones y shocks exógenos, muchos de los cuales son impredecibles. El segundo componente, en cambio, está ligado con la indefensión o la incapacidad de dichos sujetos para asumir un desastre sin incurrir en pérdidas. En estos términos, la vulnerabilidad se asocia con el riesgo de que ocurran cierto tipo de sucesos (exposición), la habilidad para soportar los impactos (resistencia), y la forma y medios para reducir y/o afrontar los daños causados (resiliencia) (Guillaumont, 1999).

Para Villagrán (2006), los acontecimientos o shocks que afectan a los individuos o colectivos pueden ser naturales (terremotos, erupciones 
volcánicas o huracanes), tecnológicos y, en diversas esferas, inducidos por los seres humanos (guerras, cambio climático, hechos económicos, etc.). De igual manera, los efectos que generan los mismos pueden estar relacionados con distintos tópicos. Como resultado, tanto las causas (shocks) como las consecuencias (pérdidas) adquieren un carácter multifacético y multidimensional (Chambers, 1989; Moser, 1998; Busso, 2001; Briguglio, 2003; Guillaumont, 2016).

Entendiendo que la vulnerabilidad puede afectar el bienestar de los individuos o colectivos en distintos planos, formas e intensidades (Busso, 2001), el estudio de la misma puede abordarse desde distintos enfoques. Desde esta perspectiva, habría varias dimensiones de vulnerabilidad, tales como: física, ambiental, económica, social, política, técnica, ideológica, ecológica, institucional, educacional, sanitaria, cultural, entre otras (Busso, 2001; Villagrán, 2006).

De acuerdo a sus características, la vulnerabilidad también puede clasificarse como coyuntural o estructural (Atkins, Mazzi y Ramlogan, 1998; Guillaumont, 1999; 2010; 2013; 2016; Briguglio, 2004; Briguglio, Cordina, Farrugia y Vella, 2006; 2009). En la vulnerabilidad coyuntural, los shocks son temporales (corto plazo) y podrían solucionarse (o exacerbarse) con medidas de intervención, que generalmente son aplicadas por los gobiernos. Entretanto, cuando la vulnerabilidad es estructural (largo plazo), las condiciones que generan la exposición tienden a ser permanentes o duraderas $\mathrm{y}$, por tanto, no dependen de la voluntad de los gobiernos (Guillaumont, 2010; 2013; 2016). De este modo, ante la ocurrencia de un shock externo, toma importancia la resiliencia, en la medida en que contribuye a reducir o a aumentar el grado de vulnerabilidad a la que ve sometido un individuo o colectivo.

En el caso de la resiliencia, esta se entiende como la capacidad que tienen los sujetos para absorber un shock exógeno y cubrirlo exitosamente, es decir, alcanzar en el menor tiempo posible el estado en que se encontraba antes de ocurrir el siniestro (Briguglio, 2003; Villagrán, 2006; Angeon y Bates, 2015; Guillaumont, 2016). De ahí que la resiliencia se asocie con la capacidad de adaptación, convirtiéndose en un mecanismo que permite reducir la vulnerabilidad (Guillaumont, 2016). Por esta razón, la resiliencia y la vulnerabilidad terminan siendo dos conceptos recíprocos (Villagrán, 2006).

Briguglio (2003; 2014) señala que la resiliencia puede ser inherente o alimentada. En el primer caso, son las condiciones existentes o adquiridas las que permiten que un sujeto o colectivo se recuperen de un daño y, en el segundo, es el Estado el que entra a intervenir a través de sus instrumentos para reducir, mitigar y/o afrontar el impacto de un determinado shock. No obstante, así como las medidas de política aplicadas por los gobiernos pueden reducir la vulnerabilidad, generando resiliencia, también pueden contribuir a exacerbarla (Briguglio et al., 2006).

A partir de los elementos citados, se colige -dentro de este estudio- que no es posible separar la vulnerabilidad de la resiliencia, entendiendo que, ante la ocurrencia de un choque externo, no solamente cuentan las características del fenómeno, sino también las capacidades que se han desarrollado para enfrentarlo.

\subsection{Tipos de vulnerabilidades}

Una vez se examinó el concepto de vulnerabilidad, en adelante, se describen los principales ámbitos desde donde se puede enfocar su estudio. Para este propósito, se sigue la clasificación propuesta por Guillaumont (2016), quien hace referencia a tres grandes áreas de macrovulnerabilidad: ambiental, social y económica. Ahora bien, debido a la utilidad que prestan para esta investigación, las dos primeras áreas se exponen desde una perspectiva muy general, ya que la mayor parte de este subepígrafe se dedicará al estudio de la vulnerabilidad económica.

\subsubsection{Vulnerabilidad social}

Desde la perspectiva social, cualquier individuo o colectivo se encuentran expuestos a una serie de fenómenos, internos y/o externos, que pueden afectar su nivel de bienestar (Busso, 2001; Briguglio, 2003). La vulnerabilidad social se explica, en primer lugar, por la situación de 
indefensión o inseguridad a la cual se enfrentan las comunidades, familias o individuos como consecuencia de un impacto traumático que se origina en cualquiera de las esferas: económica, política, ambiental, etc. En segundo lugar, la vulnerabilidad social está relacionada con la manera como estos sujetos gestionan los recursos y toman las medidas para hacer frente al impacto recibido (Moser, 1998; Busso, 2001; Pizarro, 2001; Villagrán, 2006). Para Busso (2001), la vulnerabilidad social también puede surgir como resultado del desamparo del Estado.

Para Pizarro (2001), la vulnerabilidad social, en el caso de América Latina, se explica por dos factores: en primer lugar, por los impactos provocados por el patrón de desarrollo y, en segundo lugar, por las dificultades de muchos individuos o colectivos para hacer frente y aprovechar los beneficios u oportunidades que ofrece dicho modelo. Bajo esta postura, la vulnerabilidad social es el resultado de la conjunción de una serie de factores provenientes de diferentes tópicos, cuyas consecuencias se pueden observar en variables como el desempleo, la pobreza, el hambre, etc.

Dada la situación socioeconómica en América Latina y varios países en desarrollo, sobre todo a finales de la década de los noventa e inicios de la primera década del siglo XXI, la reducción de la pobreza se convirtió en un tema central en la agenda del desarrollo (Moser, 1998). En este contexto, la vulnerabilidad terminó asociándose con la pobreza (Guillaumont, 2010). No obstante, lo que se ha señalado es que los dos conceptos, a pesar de estar relacionados, son diferentes (Chambers, 1989; Moser, 1998; Villagrán, 2006).

Para Moser (1998) y Pizarro (2001), mientras la pobreza es un concepto estático, que tiene que ver con la insuficiencia en variables como el ingreso o el consumo, la vulnerabilidad es algo dinámico y mucho más amplio. En el caso de las familias y los individuos, la capacidad de recuperación, ante los efectos negativos del cambio en el contexto, se explica por la disposición de sus activos $^{2}$ y la gestión de los mismos (Moser, 1998; Busso, 2001); no solamente por las variaciones de los flujos de ingresos. Para Moser (1998), cuando las familias cuentan con una mayor cantidad de activos, y son capaces de gestionarlos eficientemente, el grado de vulnerabilidad es menor.

\subsubsection{Vulnerabilidad ambiental}

La vulnerabilidad ambiental se define como la exposición y la sensibilidad de un sistema o comunidad frente a los efectos adversos de distintos tipos de fenómenos naturales, y la capacidad para recuperarse una vez se desencadenan (Pelling y Uitto, 2001; Smit y Wandel, 2006). Uno de los aspectos que más se resalta, dentro de estos estudios, son los daños generados por el calentamiento global y la propensión a las temperaturas extremas. Dentro de las causas subyacentes de la vulnerabilidad ambiental estarían: "el rápido crecimiento demográfico, las migraciones, la pobreza, la concentración de la población en áreas vulnerables, la baja calidad de la infraestructura, vivienda y servicios, el daño ambiental causado por la sobreexplotación de los recursos naturales y el bajo nivel de preparación para situaciones de emergencia" (CEPAL, 2000, p. 6).

Desde el punto de vista geográfico, la vulnerabilidad se origina porque hay ciertos lugares o países, en los cuales hay una mayor exposición a la ocurrencia de desastres naturales, cuyos efectos se pueden observar en el deterioro del suelo, el agotamiento de los recursos, etc. (Briguglio, 2003).

Para Briguglio (2003), la vulnerabilidad ambiental es mayor en los países en desarrollo debido a sus limitaciones en distintos tópicos -incluido el económico- y la exposición a numerosos eventos, como los ciclones, inundaciones, terremotos, erupciones volcánicas. Bajo estas consideraciones, Briguglio (2003) recalca

2 De acuerdo a este enfoque, los activos que tienen a disposición las personas son los siguientes: fuerza de trabajo, nivel de formación y estado de salud (capital humano); activos físicos y financieros, las relaciones familiares y la reciprocidad social (capital social). 
que este tipo de siniestros pueden ocurrir en cualquier país. Sin embargo, hay algunos territorios que son más propensos a sufrirlos; además, la capacidad de respuesta difiere entre unos y otros.

Desde la óptica de este estudio, la vulnerabilidad ambiental es importante en la medida en que los desastres generados por los fenómenos naturales tienen un alto impacto económico y social; afectando, en mayor medida, a los países en desarrollo y, en lo individual, a las personas más pobres (CEPAL, 2000).

\subsubsection{Vulnerabilidad económica}

Los dos tipos de macrovulnerabilidades mencionadas hasta el momento (ambiental y social) están relacionados con varios aspectos de tipo económico. Sin embargo, a pesar de su importancia, fundamentalmente por los daños que puede causar en el bienestar de las personas, en adelante, solamente se hará referencia a la vulnerabilidad económica. La decisión de acotar la revisión únicamente a la vulnerabilidad económica es relevante, pues el propósito es que se pueda hacer la comparación, entre los aportes planteados, desde los tres enfoques identificados en la introducción de este estudio.

Para el estudio de la vulnerabilidad económica, se empieza con la definición del término y luego se hace una descripción de los diferentes enfoques bajo los cuales se ha venido estudiando este concepto. Una vez se desarrollan estos dos aspectos, se pasa a hacer la revisión de las causas o fuentes de la vulnerabilidad económi$\mathrm{ca}$, para, posteriormente, ahondar en las consecuencias que esta ocasiona.

La vulnerabilidad económica se define como el grado de exposición o riesgo al que se encuentra sometido un individuo, su familia, o el país en su conjunto, ante la probabilidad de ocurrencia de shocks exógenos (Atkins et al., 1998; Guillaumont, 1999; 2013; 2016; Cordina, 2004; Briguglio, Cordina, Farrugia y Vella, 2008; 2009).

Para Guillaumont (1999; 2013; 2016),
la vulnerabilidad económica depende,

fundamentalmente, de tres tipos de componentes: el tamaño y la probabilidad de los shocks, la exposición a los shocks, y la capacidad para resistir y recuperarse de los daños ocasionados como fruto de los eventos adversos. Desde este planteamiento, la vulnerabilidad económica se explica por las características inherentes o estructurales de un país, en las cuales los gobiernos no pueden ejercer ningún tipo de influencia (dado que las perturbaciones son externas).

En los términos definidos, al inicio de este epígrafe, la política económica sería la vía a través de la cual se genera la resiliencia (coyuntural), la cual termina incidiendo positiva o negativamente en el grado de vulnerabilidad económica de un país. Así, la resiliencia económica es la capacidad de un país para absorber el efecto de los choques externos y contrarrestarlos o aprovecharlos, dependiendo de la naturaleza (negativa o positiva) de los mismos (Briguglio, 2014). Para el autor, la conjunción entre la vulnerabilidad y la resiliencia indicaría el riesgo conjunto de que una economía se vea perjudicada por los shocks externos.

Desde el punto de vista económico, el estudio de los efectos de un shock externo puede enfocarse desde una óptica individual (personas o familias) o desde un análisis de conjunto (país). De esta manera, el tema de la vulnerabilidad económica se puede abordar ya desde la perspectiva microeconómica o también desde la esfera macroeconómica (Guillaumont, 1999; Seth y Ragab, 2012). Naturalmente, los dos enfoques están relacionados. Desde lo individual, lo que se busca es explicar cómo un acontecimiento externo impacta a las personas, especialmente a las más pobres; mientras en lo macroeconómico el análisis se aborda a través de los efectos que un shock exógeno genera en el conjunto de la economía, y particularmente en el crecimiento económico (Guillaumont, 1999; Seth y Ragab, 2012).

En lo microeconómico, la vulnerabilidad se define como el riesgo o exposición al cual se enfrenta un individuo o su familia como fruto de un evento externo, el cual puede generar un resultado indeseable en su bienestar (Alwang, 
Siegel y Jorgensen, 2001; Calvo y Dercon, 2005; Dercon, 2006). El grado de vulnerabilidad depende de las características del riesgo, el tamaño del shock y la habilidad de los sujetos para responder ante estas situaciones (Alwang et al., 2001).

La vulnerabilidad microeconómica también se puede entender como una medida de la amenaza o probabilidad de que un individuo o su familia caigan en la pobreza (Alwang et al., 2001; Calvo y Dercon, 2005; Dercon, 2006; Naudé, Santos-Paulino y McGillivray, 2009; Seth y Ragab, 2012). En estos términos, Seth y Ragab (2012) advierten que un shock externo puede generar una reducción del ingreso, que daría lugar a una disminución del consumo y la riqueza, haciendo más probable que dicha familia se ubique por debajo de la línea de pobreza. En este caso, la incertidumbre juega un papel fundamental, dado que las familias pobres tienen pocos activos y un acceso limitado a los mercados de capital, generando una baja capacidad para afrontar los shocks y recuperarse de las pérdidas ocasionadas en su bienestar (Alwang et al., 2001; Hallegatte y Przyluski, 2011; Seth y Ragab, 2012). Además, se debe tener en cuenta que, en el caso de los países en desarrollo, los gobiernos tienen grandes limitaciones fiscales para afrontar estos problemas $y$, por tanto, la capacidad de ofrecer una salida a las familias es muy reducida (Seth y Ragab, 2012).

Por otra parte, Seth y Ragab (2012) advierten que la renta de las familias pobres, en los países en desarrollo, responde de manera asimétrica ante un shock, es decir, la caída que se presenta, en esta variable, durante las fases de crisis tiende a ser superior al nivel de crecimiento de la misma durante los periodos de auge. En consecuencia, cuando se presenta un shock, cuanto más grande sea la proporción de familias pobres en un país, menor será la tasa de crecimiento económico y la productividad.

Por las razones expuestas, Naudé et al. (2009) manifiestan que hay una relación de doble vía entre la vulnerabilidad y la pobreza. Para los autores, mientras la pobreza puede ser un resultado de la vulnerabilidad de ciertas familias ante la ocurrencia de un evento adverso, el hecho de que los individuos sean incapaces de responder a estos fenómenos también es una fuente de vulnerabilidad agregada. En consecuencia, la vulnerabilidad microeconómica puede generar efectos a nivel macro, $y$, a su vez, la vulnerabilidad global termina afectando a los individuos. De esta manera, aunque los conceptos de pobreza y vulnerabilidad son diferentes, al final, están muy relacionados (Alwang et al., 2001; Seth y Ragab, 2012).

Desde la perspectiva macroeconómica, la vulnerabilidad se define como el grado de exposición de un país ante la probabilidad de que ocurra un shock externo, y la capacidad para resistir y afrontar los efectos, positivos o negativos, que genera el mismo (Guillaumont, 1999). A partir de esta definición, algunas de las interrogantes que se podrían plantear serían las siguientes: ¿cuáles son los eventos adversos? (causas); ¿cómo se trasmiten los impactos de dichos shocks a la economía interna? (mecanismos de transmisión); y ¿dónde se pueden vislumbrar los daños ocasionados? (variables de impacto).

Las inquietudes relacionadas no son fáciles de resolver, y han dado lugar a distintas aportaciones, dependiendo de la perspectiva desde donde se emprenda el análisis. Según Seth y Ragab (2012), existen varios enfoques para abordar el estudio y la cuantificación de la vulnerabilidad macroeconómica (VM). A su juicio, habría dos macroperspectivas de análisis. En una primera línea, estarían los estudios en los que la vulnerabilidad macroeconómica se origina a partir de los rasgos estructurales de los países, que a su vez explicarían el mayor grado de exposición ante los shocks externos (ya sean naturales o económicos).

En una segunda clasificación, se encontrarían los estudios que asocian la vulnerabilidad con la persistencia de desequilibrios macroeconómicos, particularmente del sector financiero, que hacen que una economía sea proclive a desarrollar algún tipo de crisis; bien sea de deuda, cambiaria o bancaria. En la misma orientación, según Seth y Ragab (2012), hay otros trabajos en los cuales la vulnerabilidad macroeconómica 
se deriva no solamente de las características de funcionamiento del sistema financiero (local), sino también de los desajustes que generan las variaciones de los flujos internacionales de capital en varios indicadores macroeconómicos domésticos, que pueden llevar a una crisis financiera. Pero también podrían ocasionar una serie de efectos en la economía real. Frente a aquellas investigaciones en las cuales se relaciona la vulnerabilidad macroeconómica con los desequilibrios en los agregados reales, el problema estaría en que las mismas no ofrecen un marco conceptual lo suficientemente claro, que permita entender dicho fenómeno (Seth y Ragab, 2012).

Con el objetivo de ahondar en la discusión acerca de los diferentes enfoques bajo los cuales se puede abordar el estudio de la VM, en el siguiente sub epígrafe se presenta una breve caracterización de los mismos. También se busca encontrar algunos puntos de coincidencia, o divergencia, que permitan enriquecer la comprensión de la VM.

\subsection{Enfoques de la vulnerabilidad macroeconómica}

A diferencia de la clasificación que plantean Seth y Ragab (2012), frente a los enfoques de la VM, dentro de este estudio los mismos se agrupan en tres grandes posturas: a) la estructural, b) la que asocia la VM con la ocurrencia de episodios de crisis, y c) aquella que plantea que la VM está relacionada con la persistencia de la inestabilidad macroeconómica real. Las razones para separar el segundo enfoque propuesto por Seth y Ragab (2012) tienen que ver con que, dentro de este estudio, se considera que hay divergencias importantes entre aquellas propuestas en las cuales la vulnerabilidad simplemente se asocia con las crisis, y la visión neoestructuralista de dicho fenómeno.

\subsubsection{Vulnerabilidad macroeconómica estructural}

Los aportes realizados desde este enfoque son muy relevantes en cuanto a la conceptualización de la vulnerabilidad. Además, se resaltan los avances en el ámbito empírico, en el cual se han propuesto varias metodologías para la cuantificación de la vulnerabilidad económica (Briguglio, 1995; 2014; Guillaumont, 1999; 2010; 2016). No obstante, es importante advertir que, a pesar de varias coincidencias, las contribuciones agrupadas dentro de este enfoque discrepan en varios puntos, tanto en el plano teórico como en el empírico.

Desde estos enfoques, la vulnerabilidad económica está asociada al alto grado de exposición, al cual están sometidos algunos países, ante la probabilidad de ocurrencia de cierto tipo de fenómenos adversos, que a su vez se explica por las características estructurales que estos presentan (Briguglio, 1995; 2014; Hallegatte y Przyluski, 2011). Para los autores, la vulnerabilidad es algo indeseable, ya que incrementa la probabilidad de que un país se vea afectado por choques externos.

Las investigaciones abordadas desde la óptica estructural de la vulnerabilidad se han realizado, fundamentalmente, para los pequeños estados insulares en desarrollo (SIDS, por sus siglas en inglés); los países menos adelantados (LDCs, por sus siglas en inglés); y los países de renta baja (LICs, por sus siglas en inglés). No obstante, el análisis también ha cubierto a otros países en desarrollo, algunos emergentes y, en muy pocos casos, a los desarrollados.

Aunque los estudios acerca de la vulnerabilidad se han concentrado en cierto tipo de países, vale la pena aclarar que la existencia de dicho fenómeno no necesariamente implica un menor grado de desarrollo económico (Briguglio y Galea, 2003; Briguglio et al., 2006; Briguglio, 2014). Como ejemplo, los autores se refieren al caso de Singapur; un país que, a pesar de tener una elevada exposición ante la ocurrencia de shocks exógenos, ha conseguido altos niveles de renta per cápita. Por esta razón, los resultados alcanzados por un país no solo dependen del grado de vulnerabilidad, sino también de su capacidad de resiliencia (Briguglio, 2014).

En el plano conceptual, desde este enfoque, no solamente son importantes los shocks de 
carácter económico (comercial y financiero³), sino también los de tipo natural; recalcando el carácter multidimensional y multifacético de la vulnerabilidad (Atkins et al., 1998; Guillaumont, 1999; 2013; Briguglio, 2003). Empero, Guillaumont (2010) aclara que existe una diferencia entre los choques naturales y los económicos: mientras los primeros pueden ser de una sola vez, en el caso de los shocks económicos pueden repetirse.

Para un país, los desastres naturales son importantes en la medida en que le generan pérdidas significativas (económicas, sociales y ambientales), afectando su desempeño económico y, desde luego, el bienestar de los individuos (Briguglio et al., 2008; 2009; Baritto, 2009; Guillaumont, 2013). Por este motivo, Guillaumont (2011) justifica la necesidad de analizar la vulnerabilidad: es el principal obstáculo para el desarrollo en los países menos adelantados.

Desde la óptica temporal, la vulnerabilidad económica se puede evaluar tanto para el corto plazo (coyuntural) como para el largo plazo (estructural) (Guillaumont, 1999; 2010; 2011; 2013; 2016). Desde una perspectiva coyuntural, los estudios de vulnerabilidad económica se han direccionado hacia la identificación de riesgos de crisis, ya sea de tipo real (crecimiento económico) o financieras, bancarias o de balanza de pagos (Guillaumont, 2010; 2016). Para el autor, dicha vulnerabilidad tiene un carácter transitorio y cambiante, ya que refleja solamente las condiciones actuales de la economía, más no los elementos estructurales de la misma. En contraste, la vulnerabilidad económica estructural sería independiente de la aplicación de las medidas de intervención (voluntad política) por parte de los gobiernos (Guillaumont, 1999; 2009; 2010; 2013; 2016; Briguglio y Galea, 2003; Briguglio, 2004; 2014; Briguglio et al., 2006; 2008; 2009).

En síntesis, los planteamientos expuestos, desde estos enfoques, enfatizan en que la vulnerabilidad depende de unos factores que son permanentes, en los cuales los gobiernos no tienen ningún tipo de injerencia. Desde luego, la intervención de los gobiernos es importante, ya que puede contribuir

3 El efecto de los riesgos financieros se incorpora en Briguglio (2014). a aminorar los efectos causados por los shocks externos.

Con el propósito de profundizar en el análisis de la vulnerabilidad económica desde el enfoque estructural, en adelante, se abordan los tres elementos que la componen, que en la propuesta de Guillaumont (1999; 2010; 2013; 2016) son: exposición, choques y resiliencia. El primer factor tendría que ver con las fuentes o causas de vulnerabilidad; el segundo, con el impacto que genera dicho fenómeno sobre las variables económicas; y el tercero, muestra la capacidad de absorción o recuperación que tiene una economía ante los daños causados por un shock externo.

\section{A. Las fuentes de la VM}

A partir de los argumentos presentados hasta el momento, el grado de la vulnerabilidad macroeconómica de un país se explica por sus características inherentes o estructurales (Briguglio, 1995; 2004; 2014; Briguglio y Galea, 2003; Cordina, 2004; Briguglio et al., 2006; 2008; 2009; Guillaumont, 2009). Estos autores señalan que los países como los SIDS, LDCs y LICs están sometidos a varios factores de exposición que los hacen más vulnerables que otros. Dentro de las desventajas a las que se enfrentan dichos países se relacionan, fundamentalmente, las siguientes: tamaño de la población y del mercado, insularidad y lejanía (con respecto a los principales mercados o los de mayor tamaño), falta de dotación de recursos naturales, altos costos de transporte, mayor propensión a sufrir desastres naturales, dependencia de fuentes de financiamiento externo, alto grado de apertura económica, baja capacidad de absorción de la tecnología, alto contenido de importaciones estratégicas, ausencia de mercados competitivos, tamaño relativamente grande de la actividad del sector público, baja capacidad de diversificación de las exportaciones y el comportamiento adverso de algunas variables demográficas, entre otras 4 .

4 Guillaumont (1999), a pesar de que reconoce que la mayoría de las variables relacionadas efectivamente explican la vulnerabilidad de muchos países, anota que algunos de los factores no serían un elemento de vulnerabilidad, ya que no son acontecimientos imprevistos. 
Guillaumont (1999; 2009), quien comparte varios de los factores señalados anteriormente, clasifica los choques (fuentes de vulnerabilidad) a los que se enfrenta una economía de la siguiente manera: ambientales (cualquier tipo de desastre natural); otros shocks externos (volatilidad en los términos de intercambio, caída de la demanda externa, fluctuaciones en las tasas de interés internacionales, inestabilidad de los ingresos por exportaciones ${ }^{5}$ ); y otros (no ambientales, relacionados con factores internos, derivados por cambios políticos no previstos).

Se podrían relacionar otras variables que contribuyen a entender la exposición de un país ante un fenómeno externo, pero, como se pudo ver, las fuentes de vulnerabilidad son diversas, lo cual dificulta su identificación, conceptualización y medición. Como se verá más adelante, este hecho también hace más complejo el análisis de las consecuencias.

\section{B. Los efectos de la VM}

Hasta el momento, el análisis de la VM se ha planteado desde las causas que la generan. Como se mencionó, están ligadas a las características estructurales de determinados países. Dentro de estos enfoques, hay autores cuyo análisis se queda en este punto, entendiendo la VM simplemente como el riesgo de sufrir los efectos de un evento adverso (véase Briguglio, 1995). Sin embargo, el tratamiento de la VM también se puede abordar desde las consecuencias que dicho fenómeno ocasiona, es decir, teniendo en cuenta el impacto ocasionado sobre determinadas variables (Atkins et al., 1998; Guillaumont, 1999; Cordina, 2004). Este ejercicio también es complejo en la medida en que existen diversas variables afectadas, entendiendo que un shock externo se puede transmitir por diferentes canales, que a su vez dependen de las características del shock, el país, el momento del ciclo económico, etc. (Hallegatte y Przyluski, 2011).

5 Este autor solamente contempla los shocks comerciales y no los de tipo financiero, ya que considera que los movimientos en los flujos de capital son más coyunturales y, por tanto, inducidos por la política económica de los países.
Desde el punto de vista económico, la principal variable que refleja el impacto de los choques y desequilibrios, tanto internos como externos, es el PIB (Guillaumont, 1999; 2009; 2011; 2016; Cordina, 2004; Hallegatte y Przyluski, 2011; Dabla-Norris y Gündüz, 2014). Los aportes más importantes, en este sentido, se basan en las conclusiones obtenidas por Ramey y Ramey (1995), quienes, tomando una muestra amplia de países, encuentran una relación inversa entre la volatilidad de la producción y el crecimiento económico. Loayza y Raddatz (2007) y Hallegatte y Przyluski (2011) comparten dicha conclusión, señalando además que la volatilidad en la producción es una fuente de incertidumbre en los ciclos económicos, con un impacto mucho mayor en los países en desarrollo, y particularmente en los de renta baja (LICs).

Para Guillaumont (2006), la mayor volatilidad de la producción, generada por la exposición de la economía a los shocks externos, estaría asociada con una tasa media de crecimiento económico menor. Incluso, Dabla-Norris y Gündüz (2014) advierten que los choques externos pueden ralentizar y, por tanto, modificar la tendencia de crecimiento económico de un país; generando efectos más allá del ciclo económico. En el mismo sentido, Atkins et al. (1998) señalan que una manifestación importante de la VM es la mayor volatilidad de la producción de los países pequeños (particularmente los SIDS) con respecto a los más grandes.

Para Guillaumont (1999), los cambios recurrentes en el contexto externo tienen efectos negativos en el crecimiento económico, a través de la volatilidad de la tasa de inversión y del tipo de cambio real (inestabilidades intermedias), que a su vez se generan por tres inestabilidades primarias: los términos de intercambio, el valor real de las exportaciones y el valor agregado de los productos agrícolas. La inestabilidad de la inversión está asociada a un bajo promedio de productividad del capital, derivada de una

6 Para Guillaumont (1999; 2009), asociar la vulnerabilidad económica con los efectos negativos que genera en el crecimiento de la producción, implicaría una definición dinámica del concepto.

sociedad y economía • e-ISSN: 2389-9050 • No. 40, 2020 • pp. 99-118 
disminución de la productividad marginal de la inversión (Guillaumont, 2006). Para el autor, la inestabilidad externa también puede generar un efecto muy fuerte en las finanzas públicas, $y$, en el nivel microeconómico, los daños se pueden trasladar directamente a los productores.

En términos metodológicos, Guillaumont (2010; 2016) advierte que la volatilidad de la tasa de crecimiento económico no es una medida adecuada de vulnerabilidad. Argumenta que la inestabilidad de la producción puede deberse no solamente a factores estructurales, sino también a cambios en la política (coyunturales). A su juicio, es necesario tomar cierta cantidad de años para recoger el efecto de los factores permanentes, aunque se corre el riesgo de que la volatilidad de la producción refleje únicamente un cambio de la tendencia.

A pesar de la importancia de la volatilidad de la producción, Guillaumont (2010; 2016) insiste en que la vulnerabilidad es un fenómeno de conjunto, es decir, que no se puede evaluar analizando los choques por separado, y tomando como única variable de referencia al PIB.

A diferencia de autores como Brigluglio o Guillaumont, Cordina (2004) aborda el análisis de la VM en el marco de un modelo de crecimiento neoclásico. Resalta que una economía más vulnerable, caracterizada por una volatilidad relativamente elevada del stock de capital y de la tecnología de producción, inicialmente, muestra una tendencia a incrementar el nivel de capital per cápita y, por tanto, la producción; pero señala que el nivel de consumo per cápita sería más bajo en el estado estacionario. Dado que estos países deben dedicar una mayor cantidad de recursos para enfrentar los eventos adversos derivados de su mayor grado de vulnerabilidad, la productividad marginal del capital $\mathrm{y}$, en general, la productividad total de los factores termina siendo menor. Debido a los efectos señalados, la volatilidad del stock de capital reduciría la tasa de acumulación, afectando tanto a la producción como al consumo. Bajo estas premisas, el mayor grado de vulnerabilidad económica, que caracteriza a los países en desarrollo, terminaría afectando su crecimiento económico, pero también la convergencia con respecto a los países desarrollados (Cordina, 2004).

Aunque hasta el momento se ha destacado el vínculo entre la VM y el crecimiento del PIB por la vía de los factores estructurales, Atkins et al. (1998) señalan que dichos efectos también podrían abordarse desde el papel desempeñado por la gestión macroeconómica (factores internos). Este planteamiento se basa en las consecuencias que pueden ocasionar variables como la inflación y el déficit fiscal, cuyo impacto se va a reflejar en la inestabilidad macroeconómica y, por esta vía, en la volatilidad de la producción (véase Ramey y Ramey, 1995). Empero, cabe recordar que, dentro de estos enfoques, los efectos de la política económica se consideran transitorios, entretanto, la vulnerabilidad económica es de carácter estructural. Por esta razón, los factores internos actuarían como un mecanismo de resiliencia, que bien podrían reducir el grado de vulnerabilidad o convertirse en un factor adicional de exposición (Briguglio et al., 2006).

La resiliencia macroeconómica comprende los factores económicos, ambientales y sociales que permiten que la economía de un país se recupere más rápido de un evento externo adverso y, por tanto, sea más adaptable y menos expuesta a este tipo de shocks (Briguglio, 2003; 2004; 2014; Briguglio y Galea, 2003; Briguglio et al., 2006; 2008; 2009; Guillaumont, 2009; 2016; Baritto, 2009). Según Guillaumont (2016):

La economía más resiliente será aquella que esté menos expuesta debido a la implementación de la política. Las políticas que entrarían en esta categoría son aquellas que (i) desalientan la acumulación de grandes desequilibrios financieros externos (a menos que se utilicen para inversiones productivas que puedan financiar el reembolso de la deuda a través del tiempo), (ii) promueven la estabilidad del mercado financiero y la prudencia de sus entidades (iii) fomentan un gasto fiscal responsable y una adecuada recaudación de ingresos, (iv) facilitan una red de seguridad social que ayude a aquellos que son golpeados negativamente por el evento exógeno (v) permiten un mercado de trabajo flexible pero justo que posibilite 
la movilidad en puestos de trabajo al mismo tiempo que se minimice la explotación, y (vi) permiten controles y equilibrios apropiados con respecto a los sistemas políticos y judiciales de tal manera que se garantice la rendición de cuentas de los tomadores de decisiones (pp. 24-25).

Briguglio et al. (2006; 2008; 2009) y Briguglio (2014) señalan que el término resiliencia, en la literatura económica, se ha utilizado en tres sentidos: a) recuperarse rápidamente de un choque; b) soportar el efecto de un choque; y c) evitar el choque por completo. A partir de este planteamiento, la resiliencia de un país vendría explicada por los siguientes aspectos: estabilidad macroeconómica, eficiencia del mercado, buena gobernanza y desarrollo social (Briguglio et al., 2006; 2008; 2009). Además de los componentes señalados hasta el momento, habría otros factores que explican la resiliencia, tales como: el capital humano, la renta per cápita (Guillaumont, 2016), la política, las instituciones (Guillaumont, 2006), la flexibilidad en el mercado de trabajo, la apertura comercial (Loayza y Raddatz, 2007) y el ahorro neto de una economía (Baritto, 2009).

Al final, un país, dependiendo de su capacidad de resiliencia, a pesar de ser vulnerable, puede alcanzar un buen desempeño económico (Briguglio, 2014). Por el contrario, ante la existencia de vulnerabilidades subyacentes, y una baja resiliencia, el riesgo de que un país se vea afectado por un shock externo negativo es mayor (Hallegatte y Przyluski, 2011).

\subsubsection{Vulnerabilidad macroeconómica y crisis}

Dentro de este enfoque, el tema de la VM se empezó a tratar con mayor fuerza en el marco de los episodios de crisis a los cuales se enfrentaron varias economías emergentes durante la década de los noventa7. En los países en desarrollo, los estudios se concentraron en aquellos países que han recibido montos significativos

7 Durante este periodo, varios países se enfrentaron a situaciones de crisis. Tal es el caso de México (1994), varios países asiáticos (1995-1997), Rusia (1998), Brasil (1999), Colombia (1999), entre otros. de capital privado, cuya consecuencia ha sido una mayor sensibilidad de los mismos ante los cambios que se pueden generar en los mercados de capital (FMI, 1998). Como rasgo general, lo que buscaron dichos estudios fue identificar indicadores e instrumentos que permitieran anticipar la ocurrencia de algún tipo de crisis financiera, ya sea cambiaria, bancaria o de deuda.

Desde esta perspectiva, el origen de una crisis financiera está asociado con la acumulación de una serie de desequilibrios -ya sea en los precios de los activos, el tipo de cambio, el déficit en la cuenta corriente, en el déficit fiscal, o en el mismo sistema bancario- que en un momento se vuelven insostenibles y dan lugar a fuertes cambios en las variables macroeconómicas (FMI, 1998; Ghosh y Ghosh, 2003).

El FMI (1998) anota que las perturbaciones financieras pueden originarse por diversas causas, pero las más importantes serían: políticas macroeconómicas insostenibles, debilidades en el sistema financiero doméstico, cambios en las condiciones financieras mundiales (composición de los flujos de capital y de los vencimientos), variaciones repentinas de los términos de intercambio y de las tasas de interés mundiales, e inestabilidad política.

En cuanto a las consecuencias, las crisis pueden implicar una caída de los precios de los activos, la interrupción de los flujos de crédito internos y/o externos y, en la mayoría de los casos, una fuerte depreciación de la moneda o el abandono del sistema de fijación del tipo de cambio (en el caso de las paridades fijas o los sistemas intermedios).

A diferencia de los enfoques estructurales, desde esta perspectiva, la VM se explica por la confluencia de factores internos, puesto que los eventos externos cumplirían el papel de multiplicadores o amplificadores de los choques (FMI, 1998; Calvo, 2002; Kaminsky, Reinhart y Végh, 2004; Rocha y Moreira, 2010; Hallegatte y Przyluski, 2011; Dabla-Norris y Gündüz, 2014).

Desde el punto de vista empírico, Kaminsky et al. (1998) asocian el incremento de la VM con la emergencia de las denominadas crisis de balanza 
de pagos de segunda generación, en las cuales los países se pueden enfrentar a una serie de riesgos de profecías autocumplidas de los inversores y los efectos de contagio propios del funcionamiento del sistema financiero (véase Obstfeld, 1996). Bajo este planteamiento, la probabilidad de ocurrencia de una crisis, en muchos casos, queda desligada del comportamiento de los fundamentos macroeconómicos de un país (Obstfeld, 1996; Ffrench-Davis, 2005; Ocampo, 2012).

Desde estos enfoques, los episodios de crisis en un país pueden surgir tanto por desequilibrios internos como por fenómenos externos, que, a través del contagio y el comportamiento gregario de los inversionistas, podrían llegar a afectar sus principales agregados macroeconómicos.

Dentro de las variables utilizadas para identificar la probabilidad de ocurrencia de una crisis se relacionan las siguientes ${ }^{8}$ : exportaciones, desviaciones de la tasa de cambio real desde su tendencia, ratio entre el dinero y las reservas internacionales brutas, producción y precio de las acciones, etc. ${ }^{9}$

Dado que el objetivo de los modelos descritos es anticiparse a la ocurrencia de algún tipo de crisis, el papel que juegan los elementos estructurales como factores explicativos del desencadenamiento de dicho fenómeno es limitado (Ghosh y Ghosh, 2003); por lo cual se justifica el uso de variables coyunturales que permitan una acción más rápida por parte de las autoridades. No obstante, advierten que las debilidades estructurales hacen que un país sea más vulnerable ante la ocurrencia de una crisis financiera.

\subsubsection{Vulnerabilidad macroeconómica e inestabilidad real}

Los aportes que asocian la vulnerabilidad macroeconómica con la inestabilidad real

8 Kaminsky et al. (1998) utilizaron 105 indicadores agrupados en varios tópicos: sector externo, sector financiero, sector real, finanzas públicas, variables estructurales e institucionales, variables políticas y efectos de contagio.

9 A partir de estos planteamientos, para el caso de América Latina, Herrera y García (1999) estructuraron un sistema de alerta temprana, cuyo objetivo también es medir la vulnerabilidad macroeconómica en estos países. provienen del Estructuralismo Latinoamericano y, concretamente, del Neoestructuralismo. Así entonces, antes de pasar a exponer tanto las causas como los efectos que genera la VM, se exponen brevemente las principales contribuciones, de este enfoque, en torno al concepto de VM.

Bajo el enfoque neoestructuralista, la VM de un país está asociada tanto a los rasgos de la estructura económica como a las características del modelo de inserción comercial y financiera (CEPAL, 2001; Ocampo, 2008b). En el caso de las economías latinoamericanas, la influencia que generan los ciclos comerciales se explica por la prevalencia de un modelo de especialización productiva basado en la explotación de recursos naturales, que hace que este tipo de economías se enfrenten, recurrentemente, a las fluctuaciones de la demanda externa y de los términos de intercambio. Con relación a los flujos financieros, la vulnerabilidad macroeconómica, como se expone más adelante, está asociada a las características que muestran dichos flujos en estos países, tanto en términos de composición, volumen y volatilidad.

Ahora bien, aunque en el enfoque neoestructuralista los choques externos juegan un rol fundamental en la explicación del origen de la $\mathrm{VM}$, los factores internos también desempeñan una función muy importante. En este sentido, el argumento central es que las medidas de política económica (fiscal, monetaria, cambiaria) no contribuyen a suavizar las fluctuaciones del ciclo económico y, por el contrario, terminan amplificando cada una de sus fases. De ahí que se las defina como procíclicas (Ocampo, 2011).

Una vez esbozados los aportes fundamentales del Neoestructuralismo alrededor del concepto de $\mathrm{VM}$, en adelante, se exponen los rasgos específicos de este enfoque.

A diferencia del enfoque financiero, en el cual la VM de un país está asociada a la existencia de determinados riesgos de crisis, desde esta perspectiva lo que se busca es analizar el impacto que generan los shocks externos en las condiciones macroeconómicas de un país $\mathrm{y}$, especialmente, en 
los agregados reales. Por tanto, aunque el análisis de la emergencia de crisis financieras y/o reales no deja de ser importante, lo más significativo es la afectación que generan los cambios en los ciclos comerciales y los financieros, en las variables como el crecimiento económico y el empleo (Ocampo, 2012; Ffrench-Davis, 2015). Precisamente donde estarían los desequilibrios fundamentales de la economía de un país.

Para Ocampo (2008a), los países en desarrollo se enfrentan a una serie de restricciones y amenazas originadas en la estructura del sistema económico mundial, que dan lugar a un alto grado de exposición al comportamiento cíclico de los flujos de capital. Dichos riesgos estarían asociados con la integración segmentada de estos países en los mercados financieros internacionales, los cuales, por su propia naturaleza, muestran una serie de fallos que terminan generando efectos negativos, tanto a nivel microeconómico como macroeconómico.

Desde el punto de vista microeconómico, el incremento de la fragilidad financiera de un país se explica por el mayor grado de apertura e integración de los mercados internacionales, el desarrollo de nuevos instrumentos financieros (innovaciones financieras) y la conducta adoptada por los inversionistas (Palma, 1998; Ocampo, 2008b). Sobre el último punto, la alternancia de fases de apetito o euforia (mayor riesgo), con otras donde prima la aversión al riesgo, generan una dinámica inestable en los mercados financieros (Ocampo, 2008b; 2011).

Las fases de auge del ciclo económico tienen lugar en un contexto de exceso de optimismo que lleva a los agentes económicos a tomar riesgos muy elevados. Una vez se pierde la confianza en la economía, los inversionistas liquidan las posiciones, buscando la protección de sus activos (huida a la calidad). Es en este momento donde se generan los mayores desajustes dentro de los mercados financieros, cuyos efectos se trasladan, posteriormente, a la economía real.

En el marco de los episodios de crisis suscitados en varios países emergentes durante los años noventa, se podrían identificar al menos dos fallos en los mercados financieros: un exceso de liquidez de algunos agentes y el sobreendeudamiento de otros
(Palma, 1998). Dichos fallos tienden a reforzarse hasta que, en un momento dado, se generan determinados desequilibrios que no se pueden sostener, lo que conduce necesariamente a una crisis.

Dado el grado de integración de los mercados financieros, un episodio de crisis en un país puede generar consecuencias negativas en otros, debido al surgimiento de los efectos de contagio (Ocampo, 2008b). Fruto de dicho fenómeno, el resultado más probable desde el punto de vista macroeconómico es una interrupción repentina, y muchas veces abrupta, de los flujos financieros internacionales.

Por las razones expuestas hasta el momento, la volatilidad de la cuenta de capitales se ha convertido en la principal fuente de inestabilidad macroeconómica real para los países en desarrollo y, particularmente, para las economías latinoamericanas (Ocampo, 2008b; Ffrench-Davis, 2010; Bielschowsky, Izam y Mulder, 2011). Es en este contexto donde surge el concepto de dominancia de la balanza de pagos, que afirma que la dinámica económica de estos países en las últimas décadas ha quedado ligada a los vaivenes de los ciclos externos y, en especial, de los flujos de capital (Ocampo, 2005; 2011). El problema fundamental para las economías en desarrollo es que los flujos financieros generan efectos procíclicos sobre sus principales variables macroeconómicas (tipo de cambio, tasas de interés, crédito doméstico y precios de los activos), ocasionando una serie de desequilibrios que posteriormente tienen que corregir (Ocampo, 2005).

En la economía real, los ciclos externos terminan afectando el comportamiento de la demanda agregada (Ocampo, 2011), las decisiones de inversión, el ahorro y, desde luego, el crecimiento económico. Bajo este enfoque, la inestabilidad de la demanda agregada, de la producción y del empleo, está asociada a la dinámica de los shocks externos, representados en los movimientos de los flujos internacionales de capital y los altibajos de los términos de intercambio (Ffrench-Davis, 2010; 2015).

En términos de la demanda agregada, los efectos procíclicos generados por los ciclos externos alteran la dinámica del gasto interno. Al final, lo que se plantea es que las variaciones de la demanda 
doméstica, muy marcadas entre las fases de auge y crisis, tienden a ser mayores que las del PIB. Como consecuencia, las fluctuaciones de la demanda ocasionan un efecto contraproducente en el comportamiento de la producción, haciéndola más volátil (Ffrench-Davis, 2010). Dado el rol que juegan los factores externos, dicha volatilidad es la que genera la vulnerabilidad de este tipo de economías.

Ante los problemas mencionados, los gobiernos de los países en desarrollo, con el objetivo de reducir los riesgos a los que se enfrentan, se ven obligados a tomar decisiones de política económica que son costosas e incoherentes (Ocampo, 2008b; 2011). En este sentido, la política económica se vuelve contradictoria, porque, en vez de reducir el riesgo de estos países, termina alimentando el ciclo económico, exacerbando los auges y profundizando las caídas (Ffrench-Davis, 2005; 2015; Ocampo, 2012).

Uno de los problemas a los cuales se enfrentan estos países está en la necesidad de alcanzar la estabilidad macroeconómica a través de variables como la inflación y el déficit fiscal, lo que conlleva a que descuiden los equilibrios en las variables reales, como las tasas de interés y el tipo de cambio. Lo que ocasiona una serie de efectos negativos sobre la inversión y el crecimiento económico. A partir de los argumentos señalados, los países en desarrollo terminan reduciendo su capacidad de acción a través de la política económica, debido a que tienen que garantizar unos equilibrios macroeconómicos (nominales), que sean consecuentes con las necesidades de los agentes de mercado.

Así entonces, el fin último de este enfoque no es identificar la probabilidad de ocurrencia de una crisis. Como se ha señalado, el propósito fundamental es entender cómo una economía está expuesta a una serie de fenómenos (externos) que terminan afectando su desempeño económico; aunque no necesariamente termine con una caída de la producción o en algún tipo de crisis financiera (bancaria, cambiaria o de deuda).
En contraste con los sistemas de alerta temprana (SAT) -que son los instrumentos que se diseñaron desde el segundo enfoque señalado dentro de esta investigación- si bien es cierto que se comparte la idea de que la política económica puede ser una fuente de los desequilibrios macroeconómicos, niegan que sea la única. De hecho, en el enfoque neoestructuralista, como se ha mencionado, la influencia que ejercen los choques externos resulta fundamental.

También hay una diferencia relevante con relación al significado de la estabilidad macroeconómica. Mientras en los modelos de alerta temprana la estabilidad se mide por el comportamiento de las variables nominales, bajo el planteamiento neoestructuralista los desajustes fundamentales están en las variables como la producción, la demanda agregada, las tasas de interés y el tipo de cambio reales. Además, no solamente se concentran en elementos coyunturales, derivados del análisis del ciclo económico (corto plazo), sino que también se remiten a las características estructurales de estos países; donde estaría el origen de los desequilibrios mencionados.

\section{Conclusiones}

A partir de la revisión presentada dentro de este estudio, se pudo observar que hay grandes discrepancias frente a la conceptualización de la VM. Así, mientras los enfoques derivados de los análisis en los SIDS, LICs y los LDCs realizan una exposición amplia alrededor del concepto de vulnerabilidad y sus distintas dimensiones, no sucede lo mismo en el caso de los enfoques "financieros", ni tampoco en el Neoestructuralismo, desde los aportes de autores como Ocampo y Ffrench-Davis, entre otros.

En el primer caso, hay una preocupación por las características estructurales de los países y su propensión a sufrir los efectos derivados de los shocks exógenos, que bien pueden ser de carácter económico, fundamentalmente comercial, pero también se pueden derivar de desastres naturales. En el segundo 
y tercer grupo de enfoques, lo fundamental es lo económico.

Como fortalezas del primer enfoque, se resaltan los mayores esfuerzos por teorizar el concepto de vulnerabilidad y su medición. Sin embargo, es importante advertir algunas de sus falencias. Por un lado, no incorpora un análisis amplio de los riesgos financieros ${ }^{10}$, algo que no sería adecuado, si se tiene en cuenta que cualquier país se puede ver afectado por los ciclos de financiamiento externo y la propagación de las crisis surgidas en otros países; tal y como sucedió, a finales de los noventa, en varias economías emergentes $\mathrm{y}$, posteriormente, durante el lapso 2007-2009, en el marco de la crisis económica a la cual se vieron sometidos varios países desarrollados. Por otra parte, aunque se justifica que el análisis de la VM es de tipo estructural, se subestima el impacto que pueden generar los ciclos externos y el papel de la política económica. Tal y como lo advierten Ramey y Ramey (1995), la volatilidad de la producción en el corto plazo, que puede ocasionarse por diversos aspectos, incluidas las decisiones de los gobiernos, también puede afectar el crecimiento económico de un país.

En cuanto a los enfoques que asocian la VM de un país con la probabilidad de ocurrencia de algún tipo de crisis, que bien puede ser real o financiera, hay una serie de debilidades que es necesario advertir. En primer lugar, varios estudios han advertido acerca de su baja capacidad predictiva (Hallegatte y Przyluski, 2011; Frankel y Saravelos, 2012), y, en segundo lugar, conceptualmente, la VM no solamente tiene que ver con la identificación de este tipo de eventos. De hecho, una consecuencia de la VM es la ralentización del crecimiento económico y no necesariamente una crisis (Hallegatte y Przyluski, 2011).

Bajo la propuesta neoestructuralista, la VM está asociada con la inestabilidad real a la cual se enfrentan varias economías en desarrollo, particularmente las latinoamericanas. A partir

10 En Briguglio (2014), se incluyen algunos aspectos, pero no son de mayor relevancia. de esta investigación, se concluye que en la misma se conjugarían varias características de los dos enfoques señalados anteriormente, aunque hay una serie de elementos donde la perspectiva es diferente. Por una parte, se destaca la influencia de los aspectos estructurales en el origen y dinámica de la VM. Además, se acepta que el uso de los instrumentos de política económica en estos países, antes que contribuir a suavizar los ciclos económicos, han terminado amplificándolos.

Sin embargo, un rasgo distintivo que se esboza desde el Neoestructuralismo es la importancia de las asimetrías (productivas, macroeconómicas, financieras, de la movilidad de los factores productivos) existentes en las relaciones económicas internacionales, entre los países en desarrollo y los países desarrollados, y su influencia en el carácter de la inserción externa de los mismos (comercial y financiera). Desde este enfoque, dichos elementos resultan fundamentales para entender la VM de un país.

Por otra parte, un aspecto destacable del enfoque neoestructuralista es la relación que se plantea entre la inestabilidad macroeconómica real de un país y su grado de VM. Desde esta perspectiva, para que un país sea considerado como vulnerable no se requiere que presente un episodio de crisis. Empero, se advierte que el efecto global que genera la VM es muy relevante, y se ve reflejado en la incapacidad de sostener la senda del crecimiento económico por periodos largos.

Dentro de las deficiencias, lo que se pudo identificar a partir de esta investigación es que, en el enfoque neoestructuralista, no hay una conceptualización expresa de la VM, ni tampoco se hace un esfuerzo para su medición. Bajo estas consideraciones, una de las conclusiones obtenidas, a partir de este estudio, es precisamente la necesidad de ampliar la visión de la VM, desde la perspectiva neoestructuralista. La idea es tener mayores elementos conceptuales que permitan el análisis y sistematización de este fenómeno, sobre todo en el contexto latinoamericano. 


\section{Referencias bibliográficas}

Alwang, J., Siegel, P. B., y Jorgensen, S. L. (2001). Vulnerability: a view from different disciplines. Discussion Paper Series, 0115. Washington D. C., Estados Unidos: World Bank.

Angeon, V., y Bates, S. (2015). Reviewing composite vulnerability and resilience indexes: a sustainable approach and application. World Development, 72, 140-162. doi: 10.1016/j.worlddev.2015.02.011

Atkins, J., Mazzi, S., y Ramlogan, C. (1998). A study of the vulnerability of developing and island states: a composite index. Londres, Reino Unido: Commonwealth Secretariat.

Baritto, F. (2009). Disasters, vulnerability and resilience from a macro-economic perspective, Lessons from the empirical evidence. Recuperado de https://www.preventionweb.net/english/hyogo/gar/ background-papers/documents/Chap2/Baritto-Macroeconomic-Report.pdf

Bielschowsky, R., Izam, M., y Mulder, N. (2011). Dos estudios de la evolución del pensamiento de la CEPAL sobre la diversificación productiva y la inserción internacional (1949-2008). Santiago, Chile: CEPAL.

Briguglio, L. (1995). Small island developing states and their economic vulnerabilities. World Development, 23(9), 1615-1632. doi: 10.1016/0305-750X(95)00065-K

Briguglio, L. (2003). The vulnerability index and Small Island Developing States: A review of conceptual and methodological issues. Recuperado de https://pdfs.semanticscholar.org/ addl/0617fdablle938a236aleclocb67b32abae9.pdf

Briguglio, L. (2004). Economic vulnerability and resilience: concepts and measurements. En L. Briguglio y E. J. Kisanga (Eds.), Economic Vulnerability and Resilience of Small States (pp. 43-53). Msida, Malta: Commonwealth Secretariat and the University of Malta.

Briguglio, L. (2014). A vulnerability and resilience framework for small states. En D. Bynoe-Lewis (Eds.), Building the resilience of small states: a revised framework (pp. 1-102). Londres, Reino Unido: Commonwealth Secretariat.

Briguglio, L., y Galea, W. (2003). Updating and augmenting the economic vulnerability index. Recuperado de https://www.um.edu.mt/library/oar/handle/123456789/18371

Briguglio, L., Cordina, G., Farrugia, N., y Vella, S. (2006). Conceptualizing and measuring economic resilience. Recuperado de https://www.jstor.org/stable/pdf/j.ctt2jbk3w.ll.pdf

Briguglio, L., Cordina, G., Farrugia, N., y Vella, S. (2008). Economic Vulnerability and Resilience: Concepts and Measurements. Research Paper, 2008-55. Helsinki, Finlandia: United Nations University.

Briguglio, L., Cordina, G., Farrugia, N., y Vella, S. (2009). Economic vulnerability and resilience: concepts and measurements. Oxford Development Studies, 37(3), 229-247. doi: 10.1080/13600810903089893

Busso, G. (2001). Vulnerabilidad social: nociones e implicancias de políticas para Latinoamérica a inicios del siglo XXI. Santiago, Chile: CEPAL.

Calvo, C., y Dercon, S. (2005). Measuring individual vulnerability. Working Paper, 229. Oxford, Reino Unido: University of Oxford.

Calvo, G. (2002). Explaining sudden stop, growth collapse and BOP crisis: the case of distortionary output taxes. IMF Staff Papers, 50(special issue), 1-20. doi: 10.2307/4149912 
CEPAL -Comisión Económica para América Latina y el Caribe-. (Marzo 25-26, 2000). A matter of development: how to reduce vulnerability in the face of natural disasters. Joint ECLA-IDB paper presented at the seminar "Confronting Natural Disasters: A Matter of Development", New Orleans, Estados Unidos.

CEPAL -Comisión Económica para América Latina y el Caribe-. (2001). Crecer con estabilidad: el financiamiento del desarrollo en el nuevo contexto internacional. Bogotá, Colombia: Alfaomega.

Chambers, R. (Ed.). (1989). Vulnerability: how the poor cope. IDS Bulletin, 20(2), 1-67.

Cordina, G. (2004). Economic vulnerability and economic growth: some results from a neo-classical growth modelling approach. Journal of Economic Development, 29(2), 21-39.

Dabla-Norris, E., y Gündüz, Y. B. (2014). Exogenous shocks and growth crises in low-income countries: A vulnerability index. World Development, 59, 360-378. doi: 10.1016/j.worlddev.2014.02.001

Dercon, S. (2006). Vulnerability: a micro perspective. Working Paper, 149. Oxford, Reino Unido: Oxford University, Queen Elizabeth House (QEH). doi: 10.1596/978-0-8213-6107-8

Ffrench-Davis, R. (Ed.). (2005). Crecimiento esquivo y volatilidad financiera. Bogotá, Colombia: CEPAL y Mayol Ediciones.

Ffrench-Davis, R. (2010). Macroeconomía para el desarrollo: desde el financierismo al productivismo. Revista de la CEPAL, (102), 7-27.

Ffrench-Davis, R. (2015). Neoestructuralismo y macroeconomía para el desarrollo. En A. Bárcena y A. Prado (Eds.), Neoestructuralismo y Corrientes heterodoxas en América Latina y el Caribe a inicios del siglo XXI (pp. 119-143). Santiago, Chile: CEPAL.

FMI -Fondo Monetario Internacional-. (1998). Financial crises: characteristics and indicators of vulnerability. En World Economic Outlook, chapter IV (pp. 74-97). Washington D. C., Estados Unidos: FMI.

Frankel, J., y Saravelos, G. (2012). Can leading indicators assess country vulnerability? Evidence from the 2008-09 global financial crisis. Journal of International Economics, 87(2), 216-231. doi: 10.1016/j. jinteco.2011.12.009

Ghosh, S. R., y Ghosh, A. R. (2003). Structural vulnerabilities and currency crises. IMF Economic Review, 50(3), 481-506. doi: 10.2307/4149942

Guillaumont, P. (1999). On the economic vulnerability of low income countries. Recuperado de http:// publi.cerdi.org/ed/2001/2001.02.pdf

Guillaumont, P. (2006). Macro vulnerability in low-income countries and aid responses. En F. Bourguignon, B. Pleskovic y J. Van Der Gaag (Eds.), Securing Development in an Unstable World (pp. 65-108). Washington D. C., Estados Unidos: World Bank.

Guillaumont, P. (2009). An economic vulnerability index: its design and use for international development policy. Oxford Development Studies, 37(3), 193-228. doi: 10.1080/13600810903089901

Guillaumont, P. (2010). Assessing the economic vulnerability of small island developing states and the least developed countries. The Journal of Development Studies, 46(5), 828-854. doi: $10.1080 / 00220381003623814$ 
Guillaumont, P. (2011). The concept of structural economic vulnerability and its relevance for the identification of the Least Developed Countries and other purposes (nature, measurement and evolution). Recuperado de https://www.un.org/development/desa/dpad/wp-content/uploads/ sites/45/PDFs/CDP_Background_Paper_Series/bp2011_12.pdf

Guillaumont, P. (2013). Measuring structural vulnerability to allocate development assistance and adaptation resources. Development Policies Working Paper, 68. Recuperado de https://ideas.repec. org/p/fdi/wpaper/992.html

Guillaumont, P. (2016). Vulnerability and resilience: a conceptual framework applied to three Asian countries Bhutan, Maldives and Nepal. ADB South Asia Working Paper Series, 53. Manila, Filipinas: Asian Development Bank. doi: 10.22617/WPS179069-2

Hallegatte, S., y Przyluski, V. (2011). Managing volatility: a vulnerability exercise for Low-Income Countries. Recuperado de https://www.imf.org/external/np/pp/eng/2011/030911.pdf

Herrera, S., y García, C. (1999). User's guide to an early warning system for macroeconomic vulnerability in Latin American countries. Journal of Economics, 98(4), 463-484. doi: 10.1596/1813-9450-2233

Kaminsky, G., Lizondo, S., y Reinhart, C. M. (1998). Leading indicators of currency crises. Staff Papers - International Monetary Fund, 45(1), 1-48. doi: 10.2307/3867328

Kaminsky, G., Reinhart, C., M. y Végh, C. A. (2004). When it rains, it pours: procyclical capital flows and macroeconomic policies. NBER Macroeconomics Annual, (19), 11-53. doi: 10.3386/w10780

Loayza, N. V., y Raddatz, C. (2007). The structural determinants of external vulnerability. The World Bank Economic Review, 21(3), 359-387. doi: 10.1093/wber/lhmol8

Moser, C. (1998). The asset vulnerability framework: reassessing urban poverty reduction strategies. World development, 26(1), 1-19. doi: 10.1016/S0305-750X(97)10015-8

Naudé, W., Santos-Paulino, A. U., y McGillivray, M. (Eds.). (2009). Vulnerability in Developing Countries. Tokyo, Japón: United Nations University Press.

Obstfeld, M. (1996). Models of currency crises with self-fulfilling features. European Economic Review, 40(3-5), 1037-1047. doi: 10.1016/0014-2921(95)00111-5

Ocampo, J. A. (2005). Los caminos para superar las frustraciones de crecimiento en América Latina. En R. Ffrench-Davis (Ed.), Crecimiento esquivo y volatilidad financiera (pp. 31-56). Santiago, Chile: Mayol Ediciones.

Ocampo, J. A. (2008a). El auge económico latinoamericano. Revista de Ciencia Política, 28(1), 7-33. doi: $10.4067 /$ S0718-090X2008000100002

Ocampo, J. A. (2008b). Macroeconomic Vulnerability and Reform: Managing ProCyclical Capital Flows. Recuperado de http://citeseerx.ist.psu.edu/viewdoc/ download?doi=10.1.1.574.719\&rep=repl\&type=pdf

Ocampo, J. A. (2011). Macroeconomía para el desarrollo: políticas anticíclicas y transformación productiva. Revista de la CEPAL, (104), 13-41.

Ocampo, J. A. (2012). Balance of payments dominance: Its implications for macroeconomic policy. Recuperado de https://bibliotecadigital.fgv.br/dspace/bitstream/handle/10438/16268/ Jose $\% 20$ Antonio\% $200 \mathrm{Campo} \% 20-\% 20$ Balance $\% 20$ of\%20Payments\%20Dominance_o. pdf? sequence $=1 \&$ isAllowed $=y$ 
Palma, G. (1998). There and a half cycles of mania, panic and [asymmetric] crash: East Asia and Latin America compared. Cambridge Journal of Economics, 22(6), 789-808. doi: 10.1093/cje/22.6.789

Pelling, M., y Uitto, J. I. (2001). Small island developing states: natural disaster vulnerability and global change. Global Environmental Change, Part B, Environmental Hazards, 3(2), 49-62. doi: 10.3763/ehaz.2001.0306

Pizarro, R. (2001). La vulnerabilidad social y sus desafíos: una mirada desde América Latina. Serie Estudios Estadísticos y Prospectivos, 6. Santiago, Chile: CEPAL.

Ramey, G., y Ramey, V. (1995). Cross-country evidence on the links between volatility and growth. The American Economic Review, (85), 1138-1151. doi: 10.3386/w4959

Rocha, K., y Moreira, A. (2010). The role of domestic fundamentals on the economic vulnerability of emerging markets. Emerging Markets Review, 11(2), 173-182. doi: 10.1016/j.ememar.2009.11.004

Seth, A. y Ragab, A. (2012). Macroeconomic vulnerability in developing countries: Approaches and issues. Working Paper, 94. Recuperado de https://www.econstor.eu/bitstream/10419/71809/1/72073648X. pdf

Smit, B., y Wandel, J. (2006). Adaptation, adaptive capacity and vulnerability. Global Environmental Change, 16(3), 282-292. doi: 10.1016/j.gloenvcha.2006.03.008

Villagrán, J. C. (2006). Vulnerability: a conceptual and methodological review. Bonn, Alemania: University Research Counsel Education. 